\title{
Direct-View Multi-Point Two-Component Interferometric Rayleigh Scattering Velocimeter
}

\author{
Daniel Bivolaru ${ }^{1}$ \\ The George Washington University, Hampton VA, 23681-2199 \\ Paul M. Danehy, ${ }^{2}$ Richard L. Gaffney, Jr. ${ }^{3}$ \\ NASA Langley Research Center, Hampton VA, 23681-2199 \\ and \\ Andrew D. Cutler ${ }^{4}$ \\ The George Washington University, Newport News, VA 23602
}

\begin{abstract}
This paper describes an instantaneous velocity measurement system based on the Doppler shift of elastically scattered laser light from gas molecules (Rayleigh scattering) relative to an incident laser. The system uses a pulsed laser as the light source, direct-viewing optics to collect the scattered light, an interferometer to analyze spectrally the scattered light mixed with the incident laser light, and a CCD camera to capture the resulting interferogram. The system is capable of simultaneous, spatially $\left(\sim 0.2 \mathrm{~mm}^{3}\right)$ and temporally ( $40 \mathrm{~ns})$ resolved, multiple point measurements of two orthogonal components of flow velocity in the presence of background scattered light, acoustic noise and vibrations, and flow particulates. Measurements in a large-scale axi-symmetric Mach $1.6 \mathrm{H}_{2}$-air combustionheated jet running at a flow sensible enthalpy specific to Mach 5.5 hypersonic flight are performed to demonstrate the technique. The measurements are compared with CFD calculations using a finite-volume discretization of the Favre-averaged Navier-Stokes equations (VULCAN code).
\end{abstract}

\section{Introduction}

$\mathrm{B}$ oth experimental and computational fluid dynamics methods are widely used in the design and analysis of hypersonic air-breathing engine flow paths. Computational fluid dynamics (CFD) methods employ models that are based on statistical properties of flow turbulence. These statistical properties can be known only when multiple flow properties are measured simultaneously and instantaneously, and when the spatial and temporal scales of the turbulent fluctuations are resolved. Correlations between those properties lead to a more detailed understanding of complex flow behavior and aid in the development of multi-parameter turbulence models for computational fluid dynamics codes ${ }^{1}$.

Non-intrusive optical diagnostics systems capable of obtaining simultaneous measurements of multiple fluid properties have been used in the past to characterize high speed and combusting flows. For example, researchers have employed single-pulse coherent anti-Stokes Raman spectroscopy (CARS) ${ }^{2}$ to measure $\mathrm{H}_{2}$-air supersonic combustion flow temperature and species concentration, and Rayleigh scattering ${ }^{3}$ to probe $\mathrm{H}_{2}-\mathrm{O}_{2}$ rocket plumes to determine the average gas temperature, density, and one component of velocity.

We reported in Reference 4 a single-shot laser-based technique, consisting of the combination of an interferometric Rayleigh scattering (IRS) system ${ }^{5}$ with a dual-pump CARS system ${ }^{6}$ on a common platform. It

\footnotetext{
${ }^{1}$ Research Scientist, The George Washington University/MAE Dept., NASA LaRC/MS 493, AIAA Member.

${ }^{2}$ Research Scientist, NASA LaRC, Advanced Sensing and Optical Measurement Br./MS 493, AIAA Associate Fellow.

${ }^{3}$ Aerospace Engineer, NASA LaRC, Hypersonic Air-breathing propulsion Br./MS 168, AIAA Senior Member.

${ }^{4}$ Professor, The George Washington University/MAE Dept., 1 Old Oyster Point Road, Suite 200, AIAA Associate Fellow.
} 
provides, in gaseous media, spatially and temporally resolved simultaneous measurements of two non-orthogonal components of velocity using IRS, as well as of temperature, and species concentration $\left(\mathrm{N}_{2}, \mathrm{O}_{2}\right.$, and $\left.\mathrm{H}_{2}\right)$. Later, a mobile version of this combined system was designed for use in the large-scale test facilities at NASA Langley Research Center. ${ }^{7}$

In addition to improvements performed on the CARS system, the new interferometric Rayleigh scattering system (IRS) now has an optical configuration that facilitates the direct measurement of two orthogonal velocity components - as opposed to two non-orthogonal components as previously measured- at multiple points using a single laser pulse for probing and an interferometer for spectral analysis.

IRS $^{3,8}$ uses a narrow-band polarized laser to elastically scatter photons from molecules in the measurement volume. Receiving optics collect the Rayleigh scattered light from multiple angles, mix it with the probing laser light and analyze it spectrally with a high-resolution interferometer. The shape of the scattered light spectrum is broadened by the thermal motion of the molecules, depending on the ratio between the collision frequency (determined from the gas pressure and the shear viscosity) and the acoustic spatial frequency (determined from the resultant wave vector and the speed of sound). For a single component gas of low density, when this ratio is much smaller than one, the Rayleigh spectrum can be approximated with a Gaussian shaped spectrum. The peak of the spectrum is Doppler shifted by a frequency proportional to the bulk velocity, the spectral width is proportional to the square root of the gas temperature, and its amplitude is proportional to the gas density. In other cases, such as higher density gases, the scattering spectra include three Lorentzian-shaped peaks in the spectral shape (Rayleigh-Brillouin scattering). To measure velocity, the scattered spectrum is analyzed only for Doppler shift using the initial narrow laser spectrum as reference.

In this paper we present this new and improved IRS system, combined with the CARS system, as well as preliminary measurements of two orthogonal components of velocity performed to validate the IRS technique in a large scale facility. The tests were performed at NASA Langley Research Center's Direct Connect Supersonic Combustor Test Facility (DCSCTF) on an axisymmetric Mach $1.6 \mathrm{H}_{2}$-air combustion-heated jet flow at sensible enthalpy levels of a Mach 5.5 hypersonic flight.

\section{Apparatus}

Versions of the IRS measurement systems using direct-viewing optics to measure multiple non-orthogonal components of velocity are described in Reference 5 . The system is in close proximity ( $\sim .5$ meters) to the flow being investigated as opposed to remotely operated systems using optical fibers. ${ }^{9}$ This characteristic is desirable for increasing the efficiency of the system to analyze low-intensity scattered laser light in high-temperature combustion flows. The imaging property of the optics also reduces the influence of particulates on the measurement. To overcome the always-present incident laser background radiation, the laser wavelength drift, and interferometer property changes due to variations in temperature, noise, and vibrations, the system uses the laser light as reference in every interferogram. To minimize the error in the computed velocities, two orthogonal components of velocity are measured directly and simultaneously at multiple points. Two different implementations are discussed, the first, using one laser beam and its counter-propagating reflection. A similar arrangement was previously used by Eggins and Jakson, ${ }^{10}$ although their measurement was averaged in time. In the second implementation, two opposed optics directly collect scattered light from a single incident laser beam. ${ }^{11}$ This novel optical arrangement enabled both views of the measurement region to be passed through the same planar interferometer and viewed on the same CCD camera.

The IRS system is designed as an integral part of the combined CARS-IRS system, but it can be used independently in facilities with good optical access if a laser source is provided. A complex system in itself, the IRS system uses multiple collinear beams, separated in time, and shares the main seeded green laser (narrowband seeding is required for IRS), common optical components and laser delivery optics with the CARS system. The system, as described in Ref. 7, consists of a mobile laser system, containing the main laser, a laser energy storage system, and the laser beam delivery optics. A beam-receiving and monitoring system and the spectral analysis instrument are placed near the measurement location. The beam-receiving system, designed for the specific experiment to be performed, directs and moves the probing laser beams into the flow. The spectral analysis instrument collects, collimates, and transmits directly the Rayleigh scattered signal to a planar Fabry-Perot interferometer (solid etalon) for spectral analysis. A CCD camera records the interferograms, and a computer analyzes them for spectral content and calculates the velocity. 


\section{A. Laser System}

Referring to the optical setup of the mobile CARS-IRS laser system shown in Fig. 1, the system uses the main laser as a spectrally narrow light source. The laser is a narrowband seeded Nd:YAG @ 532 nm, FWHM $\sim=0.005 \mathrm{~cm}^{-1}, 20 \mathrm{~Hz}, 850 \mathrm{~mJ} /$ pulse, $9 \mathrm{nsec} /$ pulse, with the available energy for IRS of about $300 \mathrm{~mJ} /$ pulse. The intensity of the Rayleigh scattered light at the measurement location is directly proportional to the laser source energy. For the existing optical configuration, the gas breakdown at room temperature limits the use of laser energy in a single pulse to about $65 \mathrm{~mJ}$. To increase the Rayleigh signal and to avoid laser-induced breakdown, a fraction of the green laser energy is delayed $18 \mathrm{nsec}$ (to avoid pulse overlap) and re-injected on the same optical axis by using an energy storage system of the laser energy, which contains a pulse stretcher. The optical arrangement, described in Reference 7 is designed to obtain a burst of three highenergy collinear laser beams and multiple secondary beams of lower energy (intensity decaying exponentially). Two beam splitters $\left(\mathrm{BS}_{1}\right.$ and $\mathrm{BS}_{2}$ ) with a transmittance of $68 \%$ and $38 \%$ are used as input and output combiners respectively, and six flat mirrors $\left(\mathrm{M}_{1}\right.$ to $\left.\mathrm{M}_{6}\right)$ create three delay lines of $0.2 \mathrm{~m}$ (between beam splitters), $2.9 \mathrm{~m}$ (from the first to the second beam splitters), and $2.9 \mathrm{~m}$ (from the second to the first beam splitters) respectively. Two antireflection coated glass plates (GP) are used in conjunction with the beam splitters to compensate for the changes in optical path by refraction. At full input laser energy ( $\sim 300 \mathrm{~mJ} /$ pulse) the storage system can output up to $200 \mathrm{~mJ} /$ pulse at its output. This energy is delivered to the measurement volume with an efficiency of about $50 \%$ to $70 \%$, due to losses on additional mirrors described in the next section. As will be shown later the laser beams colinearity and overlap are monitored on the cart and at the measurement volume using a focal-plane imaging system.

\section{B. Measurement Optics}

The measurement configuration is shown in Fig. 2 together with an inset showing the top view of the scattering geometry. The collinear laser beams from the output of the laser system (laser burst) are delivered at the input of the beam receiving system using five relay mirrors (not shown) and by a pair of steering mirrors (M). Spatial location and parallelism of the beams at the input are adjusted using the combining output optics of the laser system, and the energy storage system, respectively.

In the first implementation of the system, the vertically polarized light from the laser, with wave vector $\boldsymbol{k}_{01}$, is

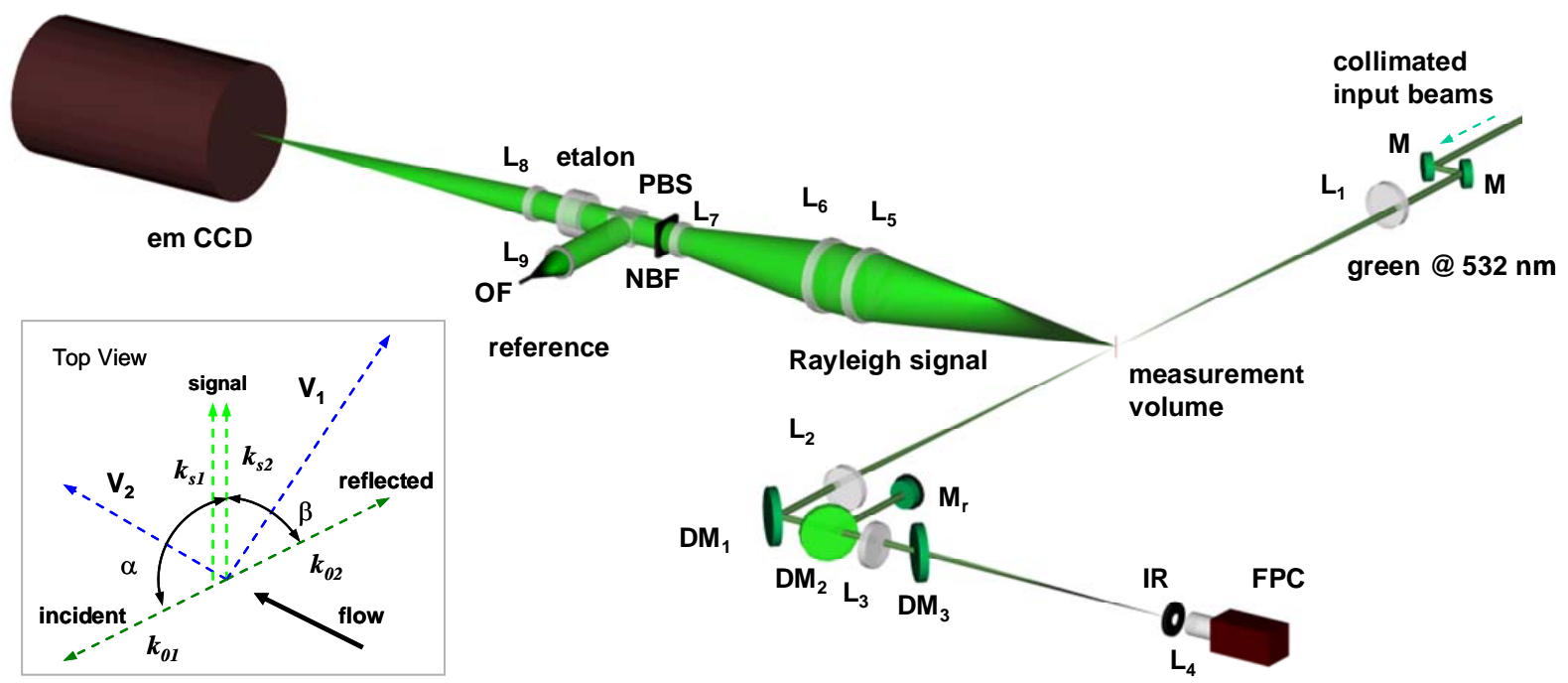

Figure 2. The measurement configuration of the IRS system. The green laser optical path is common with the CARS green laser optical path in the combined CARS-IRS system. 

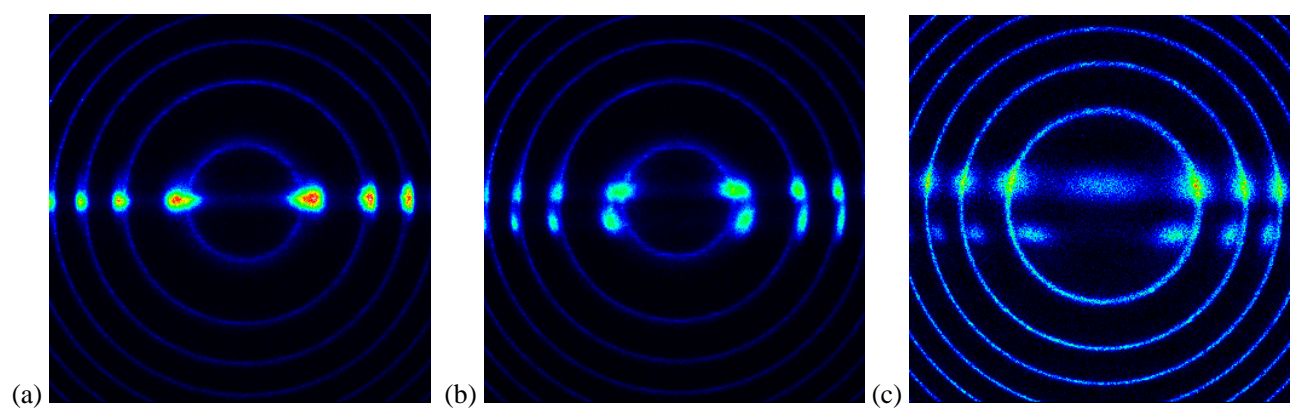

Figure 3. Spatially and temporally resolved interferograms containing reference laser frequency (concentric ring pattern) and Rayleigh scattering spectral information (horizontal pattern) used to solve directly for two orthogonal components of velocity. (a) Overlapping spectra used when the magnitude of one velocity component is much larger than the other (not the case here). (b) Separated spectra used when the magnitude of the two velocity components is unknown. The optical system uses two counter-propagating laser beams. (c) Interferogram with more pronounced reference laser frequency (concentric ring pattern) containing Rayleigh scattering spectral information (horizontal pattern) used to solve directly for two orthogonal components of velocity, v (upper), and u (lower), using a single laser beam and two collecting optics.

directed and focused using a first lens $\left(\mathrm{L}_{1}\right)$ to a measurement volume within the gas medium, providing laser illumination from one direction. Un-scattered laser light passing through the measurement volume, which is usually lost in a typical experiment, is then received by a second lens $\mathrm{L}_{2}$, collimated and redirected back toward the same lens $L_{2}$ using a retro reflective system. The reflective system necessary to filter out the CARS laser beams consists of the dichroic mirrors $\mathrm{DM}_{1}$, and $\mathrm{DM}_{2}$ (with maximum reflectivity in green) and the retro-reflective mirror $\mathrm{M}_{\mathrm{r}}$. The retroreflected laser beam of the same polarization as the initial beam is re-focused by lens $\mathrm{L}_{2}$ at the measurement volume, providing a second direction of laser illumination $\left(\boldsymbol{k}_{01}\right)$, anti-parallel with the first one. Laser light from both beams, elastically scattered in the measurement volume by the gas molecules, is then collected by lens $\mathrm{L}_{5}$ in a direction perpendicular to the laser polarization ( $\boldsymbol{k}_{\mathrm{s} 1}$, and $\boldsymbol{k}_{\mathrm{s} 2}$ directions), which maximizes the collected light. This collected light is then reduced in diameter, collimated by the lenses $\mathrm{L}_{6}$, and $\mathrm{L}_{7}$, filtered by a narrow band filter (NBF) to remove the scattered light from the yellow and the red CARS beams (when combined with CARS) and reduce the combusting flow luminosity, combined with a fraction of the incident laser light using a beam combiner (PBS) and passed through a planar interferometer (solid etalon) for spectral analysis. The interference fringe pattern formed by lens $\mathrm{L}_{8}$ at the output of the etalon is recorded by an electron multiplication gain CCD camera (emCCD) at $20 \mathrm{~Hz}$, matching the laser pulse repetition rate. The incident laser light used for reference is delivered to the system using the optical fiber OF and the collimating lens $L_{9}$. The angles defined by the incident wave vectors $\boldsymbol{k}_{01}$ and $\boldsymbol{k}_{02}$ and the scattered wave vectors $\boldsymbol{k}_{\mathrm{s} 1}$ and $\boldsymbol{k}_{\mathrm{s} 2}$ are $\alpha$ (backward scattering) and $\beta$ (forward scattering), with the corresponding velocity components $\mathrm{V}_{1}$ and $\mathrm{V}_{2}$ being measured. Since the probing laser beams are anti-parallel, the two components of velocity being measured are orthogonal.

Several interferograms obtained in high speed jet flows are shown in Fig. 3. In Figs. 3(a) and 3(b) the jet temperature is about $175 \mathrm{~K}$ and $\mathrm{M}=1.7$ and the first implementation of the IRS system is used. The resulting recorded images contain the scattered light spectral information (horizontal patterns) and the laser frequency (concentric ring pattern) used as reference. The horizontal pattern is the image of the laser beam (spatially resolved) spectrally filtered by the etalon.

Fine adjustments of the position of the beams at the focus of lens $\mathrm{L}_{1}$, are performed through the use of the pair of adjustable narrowband dichroic mirrors $\mathrm{M}$ as well as by adjusting the retro reflector mirror $\mathrm{M}_{\mathrm{r}}$. However, if the mirror $\mathrm{M}_{\mathrm{r}}$ is oriented perpendicular to the optical axis, the spectra of scattered light from both collection directions overlap on the interferogram as in Fig. 3(a). Then, the interference pattern corresponding to two different velocity components cannot be separated spatially. This ambiguity can be removed if the experiment is designed so that one measured velocity component is always much bigger than the other (for example on the axis of a high-speed jet, the axial velocity component is generally much larger than the radial component). An alternative way to remove this ambiguity is to separate the retroreflected beam from the original beam at the focus by slightly changing the angle of the retro reflector $M_{r}$. The new image contains two parallel patterns situated symmetrically around the center of the interferogram, as shown in the example interferogram of Fig. 3(b). Each of the horizontal fringe patterns in the image plane represents a velocity measurement direction, e.g. the axial (top) and the radial (bottom) velocity components. The concentric ring pattern is the interferogram produced by the source laser that is used as the 
reference frequency. The interferograms were obtained in high speed flows with a gas static temperature of about $175 \mathrm{~K}(\mathrm{M}=1.7)$ for the interferograms shown in Fig. 3(a) and Fig. 3(b), and in a $\mathrm{H}_{2}$-air combustion flow of about $1200 \mathrm{~K}(\mathrm{M}=1.6)$ for the interferogram shown in Fig. 3(c).

In the second implementation which uses laser light from one incident direction, the Rayleigh scattered light is collected from two parallel but opposite directions, combined in a single signal beam using a retro-reflector, and analyzed spectrally with one interferometer as described in detail in Reference 11. In Fig. 3(c), the interferogram (which has similar spectral content to that shown in Fig. 3(b)) displays an inward displaced Doppler shifted Rayleigh spectra for a positive velocity component in contrast to the spectra shown in Fig. 3(b) which displays an outward displaced Doppler shift. In both implementations we used for spectral analysis a solid etalon with a free spectral range (FSR) of $8.33 \mathrm{GHz}$.

\section{Laser Beams Monitoring Optics}

To maximize the Rayleigh signal, to maintain optimum setup of the energy storage system, and to monitor the influence of vibrations on the laser burst, the laser beams are monitored in real time using a focal plane imaging system (CRB-View). ${ }^{12}$ This system allows the overlap of the multiple green beams to be optimized. The CRB-View does not waste input beam energy to generate a real image of the beams' profile. This is extremely important for measurements at high temperature, when the desired increase in signal is achieved by increasing the laser energy.

In the particular arrangement shown in Fig. 2, the output beams, which have already passed through the measurement volume, are reflected by the dichroic mirror $\mathrm{DM}_{1}$ toward the lens $\mathrm{L}_{3}$ (identical in focal length to $\mathrm{L}_{1}$ ), which then refocuses them. The beams reflected by the mirror $\mathrm{DM}_{2}$ are directed toward the retro-reflector $\mathrm{M}_{\mathrm{r}}$ for IRS use. A third dichroic mirror $\mathrm{DM}_{3}$, centered at $532 \mathrm{~nm}$ with maximum reflectivity at $45 \mathrm{deg}$, further reduces the beam's energy. Angular tuning of this mirror around the maximum reflectivity position allows adjusting the transmitted energy toward the focal plane camera (FPC). Depending on the beam energy, neutral density filters (not shown) can be introduced in the optical path to reduce the beams' intensity before reaching the camera. A microscope objective lens $\mathrm{L}_{4}$ (48 $\mathrm{mm}$, chosen for the desired magnification), is placed near the focal region of lens $\mathrm{L}_{3}$ to form a magnified image of the crossing beams region on the FPC. The iris, IR, is used to remove stray light and secondary images of the beams that are formed by the dichroic mirrors. Different planes near the crossing point of the beams can be observed as the lens $\mathrm{L}_{4}$ and the camera are simultaneously translated parallel to the optical axis.

\section{Interferogram Processing}

Generally, for an axisymmetric optical setup using a uniform light source and an etalon as a spectral filter, the transmission of scattered light through the etalon is a function of the radial distance from the center $r$ of the interferogram at any given angular location $\theta$ (two dimensional Airy function). Therefore, to obtain as much information as possible from the interferograms recorded digitally on a CCD camera of rectangular symmetry ( $\mathrm{x}$ columns by $y$-lines), a coordinate transformation of the intensity distribution $\mathrm{I}(x, y)$ is necessary. The interferogram processing methodology involves the detection, extraction, and linearization of spectra from this type of interferogram (of circular symmetry) ${ }^{5}$ and consists of 6 main steps, applied to each interferogram:

In step 1$)$, the center of the interferogram circular pattern $\left(x_{0}, y_{0}\right)$ is found in the $(x, y)$ coordinate system by averaging a few lines of pixels in the vertical and horizontal direction using arbitrary (guessed) coordinates of the center. Fitting of these spectra determine the center coordinates of the interferogram with sub-pixel resolution.

In step 2), particular to the implementation using two laser beams imaged as parallel lines to the $\mathrm{x}$-axis around the center of interferogram, the locations of the laser beams in the interferogram are detected by binning all pixel columns in one averaged data. The resulting distribution approximates the intensity distribution across the laser beams, being separated by the beams setup distance on a background noise depending on the reference intensity the background noise usually being much smaller than the laser intensity. The maxima of these signal profiles give the location $y_{1}$ and $y_{2}$ of the beams with respect to the center coordinate of the interferogram $y_{0}$. Usually this step is skipped in experiments performed at laboratory conditions when the beams locations are known and are spatially stable. As will be shown later, this step is required for measurements in large scale facilities due to laser beam spatial instability due to turbulence and/or beam steering.

In step 3), using the center of the interferogram pattern $\left(x_{0}, y_{0}\right)$ found in step 1$)$, the interferogram image is remapped into a new coordinate system to facilitate the extraction of as much information as possible. We use a transformation to either $(r-\theta)$ or $(r-y)$ coordinate systems. The $(r-\theta)$ coordinate transformation has the advantage of re-mapping the interferogram in a single new image by varying $\theta$ from 0 to $360^{\circ}$. The $(r-y)$ transformation is used only in to the implementation using a laser beam imaged as a parallel line to the $\mathrm{x}$ axis (a few pixels wide) near the interferogram center, is applicable for one quadrant of the interferogram at one time. This is due to the fact that 
(a)

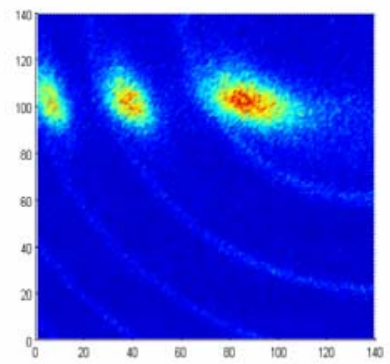

(b)

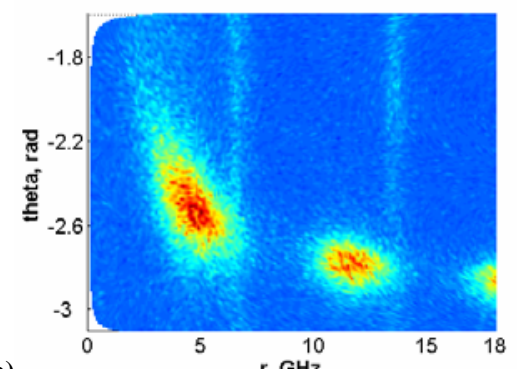

linearized interferograms containing the reference laser and the Rayleigh spectra.

along any pixel line of constant $y$ (parallel with the $x$-axis) there are two distinct points that have the same value of $r$ on each side of the $x=0$ column.

Because the mapping generates non-uniform grid data, in step 4), a two dimensional cubic spline fitting technique is applied to the new non-evenly spaced data to generate $\mathrm{I}_{\theta}(r, \theta)$, or $\mathrm{I}_{\mathrm{y}}(r, y)$, evenly-spaced data in the new coordinate system.

In step 5) the fringe spacing is linearized using the relation $R_{p+1}=\left(R_{p}^{2}+2 f 2 / k\right) 1 / 2$ between two consecutive fringe orders (ring patterns) of the interferogram, where $R$ is the radius of the ring (in interference fringe order units), $p$ is the fringe number, $k$ is the order of interference, and $f$ is the focal length of the fringe forming lens. Polynomial interpolation is used to obtain the function between fringe orders using reference spectra containing multiple orders of interference (usually more than 9 orders).

In the final step, step 6$)$, the average spectra $\mathrm{I}_{\text {ave }}(r)$ necessary for calculations, is obtained by averaging data from the respective mapping at constant $r$ in a range of $\theta$, or $y$, containing the signal after determining its location from step 2).

As an example, the result of applying these steps to one quadrant of the interferogram shown in Fig. 3(c) is shown

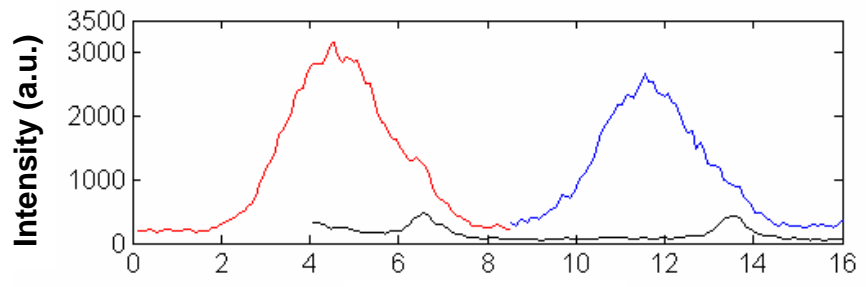

(a)

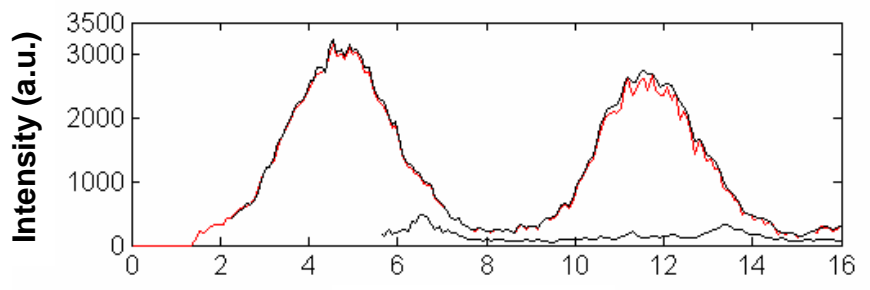

(b)

Radius (a.u.)

Figure 5. Linearized single-shot spectra of Rayleigh scattered light extracted from interferograms. (a), spectra averaged separately by $\theta$, at two points. (b), spectra "averaged by y" (black) and "averaged by $\theta$ limited by y" (red) obtained simultaneously at two points. The low amplitude spectra common to both figures are the reference spectra used to determine the spectral Doppler shift of the scattered light. in Fig. 4. The original interferogram $\mathrm{I}(x$, $y$ ) for this quadrant is shown in Fig. 4(a). Figure 4(b) represents the transformed and linearized interferogram, $\mathrm{I}_{\theta}(r, \theta)$, in the $(r, \theta)$ plane. The linearized $\mathrm{I}_{\mathrm{y}}(r, y)$ transformation result is shown in Fig. 4(c). The vertical pattern of parallel lines is the transformed concentric ring pattern of the reference. The horizontal pattern is the Rayleigh spectra corresponding to one component of velocity $\left(\mathrm{V}_{2}=\mathrm{u}\right.$ along the jet centerline) being measured at multiple spatial locations (three points in this figure).

In our particular case of a narrow laser beam source imaged parallel to the $\mathrm{x}$-axis of the interferogram pattern and located near its center, the averaging of $\mathrm{I}_{\theta}(r, \theta)$ over a number of rows ('average over $\theta^{\prime}$ ), that is smaller than the number of rows covered by one beam size may produce distorted $I_{\text {ave }}(\mathrm{r})$ spectra. This may result in systematic errors in measuring simultaneously at multiple points. One option is to extract the spectra separately for each fringe order (each measuring point), and average 
over angles containing only the signal beam. The fitting is performed only for the respective fringe order, neglecting the rest. The disadvantage of not using the remaining orders of interference is compensated by the fact that the reference spectra is always present, which is crucial to finding the Doppler shift with minimum errors. Figure 5(a) shows two plots obtained by averaging the region containing the source (Fig. 4(b)) around the first fringe order (red trace) and second order (blue trace). Clearly, multiple averaging shows, in both cases a similar fine structure around the maximum of the Rayleigh spectra. The low amplitude spectra in Fig. 5 are the reference spectra (laser wavelength markers).

The average spectra $I_{\text {ave }}(\mathrm{r})$ can also be obtained by the averaging of data over the y limits of the laser beam in either, $\mathrm{I}_{\mathrm{y}}(r, y)$ or $\mathrm{I}_{\theta}(r, \theta)$ mapping,

$$
I_{a v e}(r)=\frac{1}{2 m+1} \sum_{y=y_{1}-m}^{y_{1}+m} I_{y}(r, y) \cong \frac{1}{2 m+1} \sum_{y=y_{1}-m}^{y_{1}+m} I_{\theta}\left(r, \sin ^{-1}(y / r)\right),
$$

where $y_{1}$ is the location of the center of the beam and $m$ is an integer defining the limits of the "average over $y$ " across the beam. The average over $\theta$ is restricted by the y bounds as shown in the second expression. The average is approximate since the angle $\theta$ calculated from the $y$ bounds might not have an exact match in the $\mathrm{I}_{\theta}(r, \theta)$ mapping. We call this average the "average over $\theta$ limited by $y$ ". In Fig. 5(b), the "averaged over y" spectra extracted from the $\mathrm{I}_{\mathrm{y}}(r, y)$ mapping (black trace) are compared with the "average over $\theta$ limited by $y$ " spectra extracted from the $\mathrm{I}_{\theta}(r, \theta)$ mapping (red trace). The "averaging over y" better preserves the fine structure of the spectra than the "average over $\theta$ limited by y" although the fine structure of the first spectra appear to be slightly different as compared with the spectra shown in Fig. 5(a). The second spectral peak shows much better the symmetric fine structure of the Rayleigh spectra used in this example. The small discrepancies between the "average over y" and "average over $\theta$ limited by y" versus local "average over $\theta$ " are compensated by the shorter routine run-time gained during processing of a large number of spectra.

The calculations of the Doppler shift shown in Section V. of this paper used the "average over y" spectra, $\mathrm{I}_{\text {ave }}(\mathrm{r})$, of the transformed interferogram $\mathrm{I}_{\mathrm{y}}(r, y)$.

\section{Facility Setup}

The measurement technique has been demonstrated in an axially-symmetric free jet generated by a convergentdivergent nozzle fixed horizontally in the DCSCTF facility at NASA Langley Research Center. The convergentdivergent nozzle made of copper is designed for Mach 1.6 flow with an exit diameter of $63.5 \mathrm{~mm}$ and is operated at high enthalpy, the equivalent of a Mach 5.5 hypersonic flight. This type of geometry provides good optical access, which is required for the Rayleigh technique, and the flow symmetry can be used to minimize the number of spatial points required to describe the flow field. The flow consists of a central hot jet of $\mathrm{H}_{2}$-air combustion products and the facility is able to provide a surrounding annular co-flow jet of unheated gas. The hot jet gas is vitiated air (the products of combustion of $\mathrm{H}_{2}$, air and $\mathrm{O}_{2}$ with $23.14 \%$ by mass un-reacted $\mathrm{O}_{2}$ ). By varying the flows of reactants entering the burner, the temperature of the jet exiting the nozzle can be varied. The presented measurements are performed in the absence of the co-flow.

The measurement optics surround the test flow to be investigated. Two linear translation stages powered by servomotors provide the means of translating the measurement location up to $0.3 \mathrm{~m} \mathrm{x} 0.6 \mathrm{~m}$ area in the radial (y) and axial (x) flow directions with the origin situated axially in the nozzle exit plane. The overall positioning accuracy of the whole structure is approximately $\pm 400 \mu \mathrm{m}$. The user interface to the translation stage is through a personal computer connected to the motor controller. The program controls the motors positioning using a text input file of multiple positions to be measured and a dwell time at each location. When executed, an output file is generated with the position data sent by the motor encoders and the trigger index of the laser Q-switch pulse. This provides the means to correlate the acquired data to the specific coordinates measured in the flow.

The laser system is placed in a room located under the facility on a movable cart ${ }^{7}$ containing the main laser, the energy storage system, the auxiliary lasers, and optics and devices used for the CARS system. The total optical path between the main laser output and the measurement location is about $15 \mathrm{~m}$ ( 7 meters before it leaves the cart). The movable cart measures approximately $2.2 \mathrm{~m} \mathrm{x} 1 \mathrm{~m} \mathrm{x} 1.8 \mathrm{~m}$ and weighs $900 \mathrm{~kg}$. 
During the facility run of $60 \mathrm{sec}$ the jet was probed for $50 \mathrm{sec}$ at four selected locations (200 data points each), recording 1000 interferograms (320 x320 pixels) at the laser repetition rate of $20 \mathrm{~Hz}$. The remaining roughly 200 data points were acquired while moving between locations.

\section{Numerical Simulation of the Flow Field}

A numerical simulation of the jet flow without co-flow was performed using the VULCAN CFD code. ${ }^{13}$ This code is able to simulate finite-rate chemically reacting flow-fields using a finite-volume discretization of the Favreaveraged Navier-Stokes equations. The inviscid fluxes were computed using Edward's low dissipation flux split scheme in conjunction with a $3^{\text {rd }}$ order MUSCL stencil and a limiter by van Leer. Turbulence was modeled using the Wilcox k-omega model. In order to include the effects of turbulence generated in the facility combustor, the flow in the combustor was solved in addition to the external flow. The combustor grid had 30,720 points and the external grid had 473,088 points. A slight pressure gradient was applied across the external domain to mimic the flow through the test cell and to aid convergence by convecting starting vortices downstream and out of the solution domain. To reduce the computational time, the chemical composition of the combustor flow corresponds to a postcombustion composition. The chemical composition was assumed to be $\mathrm{N}_{2}(0.611781 / \mathrm{mol}), \mathrm{O}_{2}(0.19601 / \mathrm{mol}), \mathrm{H}_{2} \mathrm{O}$ $(0.17857 / \mathrm{mol})$, and $\operatorname{Ar}(0.00761 / \mathrm{mol})$. The total temperature and pressure in the combustor were $1468.7 \mathrm{~K}$ and 4.217 atm. Wall functions were used to reduce grid spacing requirements near walls. An isothermal boundary condition with a wall temperature of $330 \mathrm{~K}$ was used for all surfaces. The solutions were computed solving the combustor flow-field first followed by the external flow-field. The combustor grid was converged 5.5 orders of magnitude and the external grid was converged 6.4 orders of magnitude.

Figure 6 shows the axial (top half) and radial (bottom half) velocity distribution for the first 10 nozzle diameters

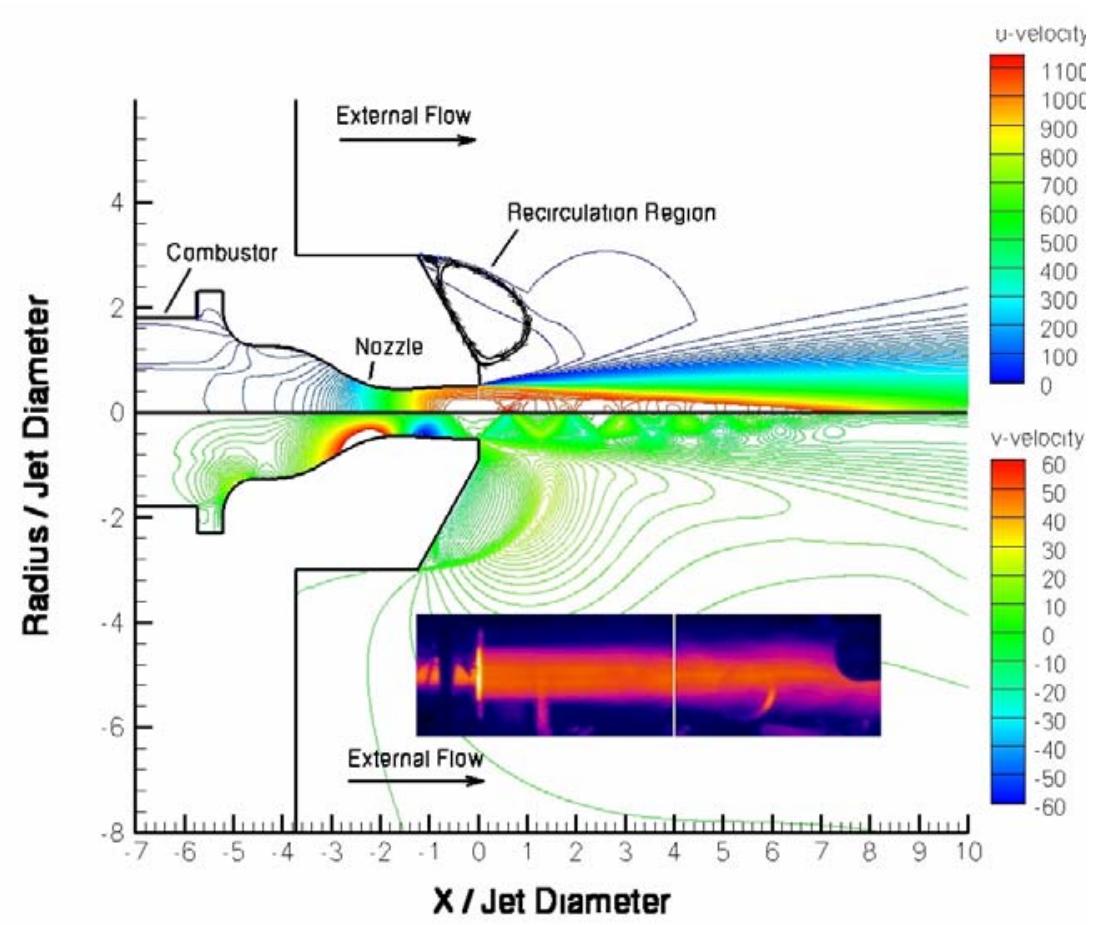

Figure 6. Axial (top half) and radial (bottom half) velocity contours of the flow using the VULCAN numerical simulation. The CFD code simulates finite-rate chemically reacting flow-fields using a finite volume discretization of the Favre-averaged Navier-Stokes equations. The conditions are as for a slightly under-expanded case. An infrared image of the jet in the $3.5 \mu \mathrm{m}$ band is inserted to scale in the bottom part of the figure. The flow is from left to right. downstream of the nozzle exit. The flow is from left to right. Within the supersonic core flow, the calculated axial velocity $\mathrm{u}$ fluctuates between 1040 and 1140 meters per second along the jet centerline. An infrared image of the jet in the $3.5 \mu \mathrm{m}$ wavelength band is shown in the inset of the figure. In a false color scale the image shows the high temperature core of the jet. The dark and bright objects in the field of view are the nozzle on the left and the optical components. At a combustor pressure of $4.217 \mathrm{~atm}$, the Mach 1.6 nozzle is slightly underexpanded $(1.046 \mathrm{~atm})$. As a result a very weak expansion fan is generated at the lip of the nozzle exit as shown in the velocity contours in Fig. 6. This expansion wave interacts with the shear layer creating a series of expansion waves and shock waves, which continue downstream a short distance. Eventually the shear layer converges on the centerline and the flow becomes entirely subsonic. 


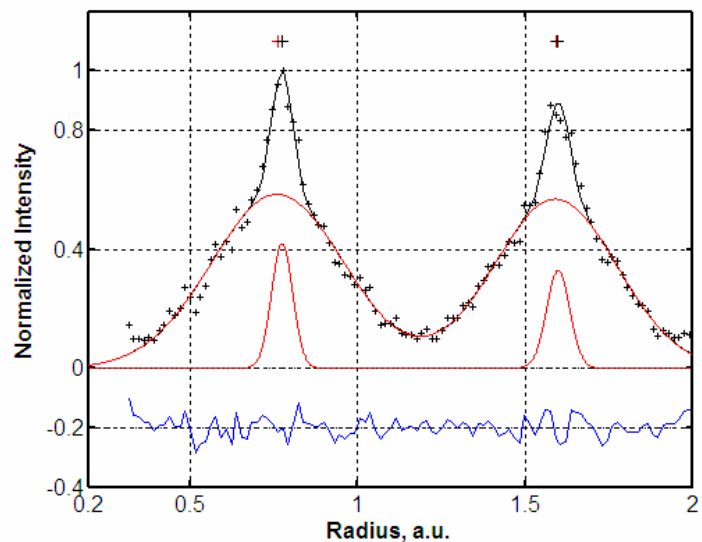

(a)

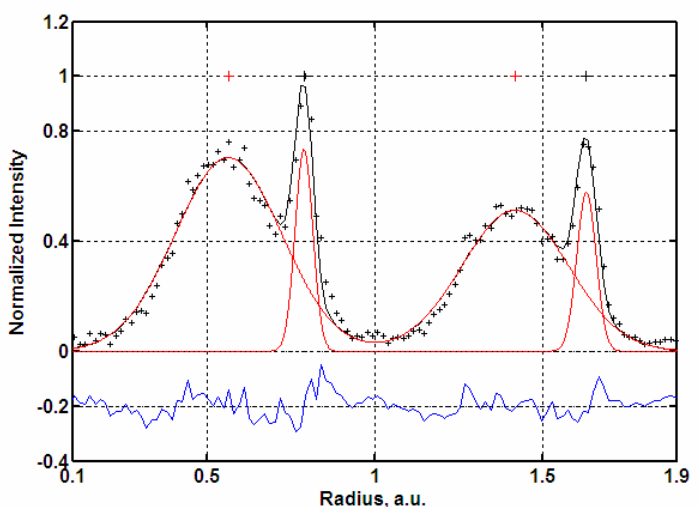

(b)

Figure 6. Single laser pulse spectra of interferometric Rayleigh scattering collected simultaneously at two spatial locations $\mathbf{0 . 4}$ $\mathrm{mm}$ apart. Each plot, (a) radial, and (b) axial, shows the experimental data (black cross), the theoretical fit (black line), and the residual between them (blue). The fit functions are Gaussian functions (red lines) with the narrow peak being at the laser frequency. Data obtained in a large-scale Mach 1.6 $\mathrm{H}_{2}$-air combustion-heated supersonic jet. In (b), the spectral Doppler shifts are $2.226 \mathrm{GHz}$ and $2.099 \mathrm{GHz}$, respectively.

of $60^{\circ}$ (forward scattering) to measure the axial component of system with a collecting geometry having an angle measure the radial component of velocity.

Spatially resolved single-pulse spectra at two-points obtained in the flow with the IRS system are shown in Fig. 6(a) for the radial component and Fig. 6(b) for the axial component, respectively. Each figure shows the average measured spectra (cross symbols), the Gaussian fit (black line), the residual between them (blue line), and the resulting signal and reference Gaussian functions extracted from the fit (red lines - the narrowest being the reference). Spectra were extracted from interferograms similar to those shown in Fig. 3(c), obtained, simultaneously with the CARS spectra (not shown). The measurement volume defined by the IRS system magnification is a number of four non-evenly spaced points of about $0.2 \mathrm{~mm}^{3}$ each, resembling the laser beam pattern seen in each interferogram. For the current configuration, these non-evenly spaced points are distributed along about $1.6 \mathrm{~mm}$ of the laser beam focus. The furthest points are situated about $0.8 \mathrm{~mm}$ apart, with the closest at about $0.4 \mathrm{~mm}$ apart. Similarly, there are two locations on the right side of the interferogram, up to a total of four resolvable measurement points in each image (expandable to six points). The total beam energy at the measurement volume was about 110 $\mathrm{mJ}$ per laser burst.

In contrast to the almost zero Doppler shifts of the spectra shown in Fig. 6(a) for the radial component of velocity, the spectra shown in Fig. 6(b) for the axial component shows a Doppler shift of about $2.2 \mathrm{GHz}$ for the first measurement point and $2.1 \mathrm{GHz}$ for the second. The spectra in Fig. 6(a), is also broader than the spectra in Fig. 6(b) 
Table 1. Simultaneous measurements of two velocity components at two spatial locations near the jet centerline slightly off axis at $(x, y) / d=(0.879,0.175)$ compared with the CFD calculations.

\begin{tabular}{|c|c|c|c|c|c|}
\hline Run 69 & $\mathbf{V}_{\mathbf{m}}(\mathbf{m} / \mathbf{s e c})$ & $\boldsymbol{\sigma}_{\mathbf{V m}}$ & $\left.\mathbf{V}_{\mathbf{m}}\right|_{\text {ave }}(\mathbf{m} / \mathbf{s e c})$ & $\mathbf{n}_{\text {ave }} / \mathbf{2 0 0}$ & $\mathbf{V}_{\mathbf{C F D}}(\mathbf{m} / \mathbf{s e c})$ \\
\hline $\boldsymbol{u}_{\mathbf{1}}$ & 1186.8 & 48.7 & 1136.9 & 170 & 1109.2 \\
\cline { 1 - 3 } $\boldsymbol{u}_{\mathbf{2}}$ & 1067.1 & 47.9 & & & \\
\hline $\boldsymbol{v}_{\mathbf{1}}$ & 85.8 & 36.0 & 67.8 & 190 & 15.1 \\
\cline { 1 - 3 } $\boldsymbol{v}_{\mathbf{2}}$ & 49.9 & 37.3 & & & \\
\hline
\end{tabular}

Table 2. Simultaneous measurements of two velocity components at two spatial locations on the jet centerline at $(x, y) / d=(0.879,0.0)$ compared with the CFD calculations.

\begin{tabular}{|c|c|c|c|c|c|}
\hline Run 62 & $\mathbf{V}_{\mathbf{m}}(\mathbf{m} / \mathbf{s e c})$ & $\boldsymbol{\sigma}_{\mathbf{V m}}$ & $\left.\mathbf{V}_{\mathbf{m}}\right|_{\text {ave }}(\mathbf{m} / \mathbf{s e c})$ & $\mathbf{n}_{\text {ave }} / \mathbf{2 0 0}$ & $\mathbf{V}_{\mathbf{C F D}}(\mathbf{m} / \mathbf{s e c})$ \\
\hline $\boldsymbol{u}_{\mathbf{1}}$ & 1139.2 & 47.1 & 1104.7 & 93 & 1101.5 \\
\cline { 1 - 3 } $\boldsymbol{u}_{\mathbf{2}}$ & 1070.2 & 53.6 & & & \\
\hline $\boldsymbol{v}_{\mathbf{1}}$ & 32.9 & 46.7 & -3.3 & 147 & 0.0 \\
\cline { 1 - 3 } $\boldsymbol{v}_{\mathbf{2}}$ & -39.6 & 48.3 & & & \\
\hline
\end{tabular}

(the valley between the two peaks in Fig. 6(b) is deeper) due to the sensitivity of Rayleigh scattering to the angle of collection: back scattering has a larger magnitude of the scattering vector than does forward scattering.

A series of 200 interferograms (200 x 4 x 4 spectra) were processed in this way at two measurement locations near the jet exit. The total temperature and total pressure in the stagnation chamber were $3.986 \mathrm{~atm}$ and $\quad 1228.9 \quad \mathrm{~K}$ respectively for the first location (Run 69) and $3.966 \mathrm{~atm}$ and $1226.1 \mathrm{~K}$ respectively for the second location (Run 62 ). Both locations are situated slightly under one nozzle exit diameter (d) downstream of the nozzle exit at $\mathrm{x} / \mathrm{d}=0.879$ with one on the axis and the other slightly off-axis at $\mathrm{y} / \mathrm{d}=0.175$. The results are shown in Fig. 7 . For comparison, an average of velocity at the respective location was extracted from the CFD data and is shown in the figure as a continuous line. The results are summarized in Table 1 and Table 2 along with the measured data, the standard deviation of the measurement, and the number of measurements used for the average from a maximum of the 200 measured spectra. The measurement uncertainty in the scattering angle of about $1^{0}$ (relative to the maximum velocity u being measured) contributes to a systematic error in calculating the velocity components of about $1.5 \%$ for the axial u component, and about $0.5 \%$ for the radial v component.

Considering that the effect of turbulence is the lowest on the axis of the jet, the velocity fluctuations of 35 to 55 $\mathrm{m} / \mathrm{sec}$ (one standard deviation) shown in the plot are mainly attributed to the errors introduced by the fitting of low

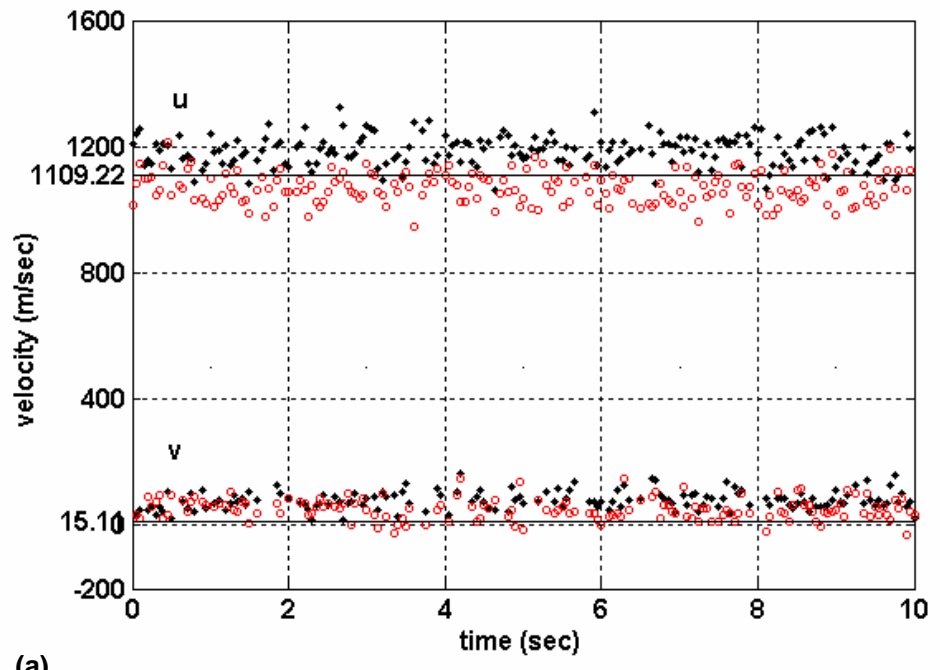

(a)

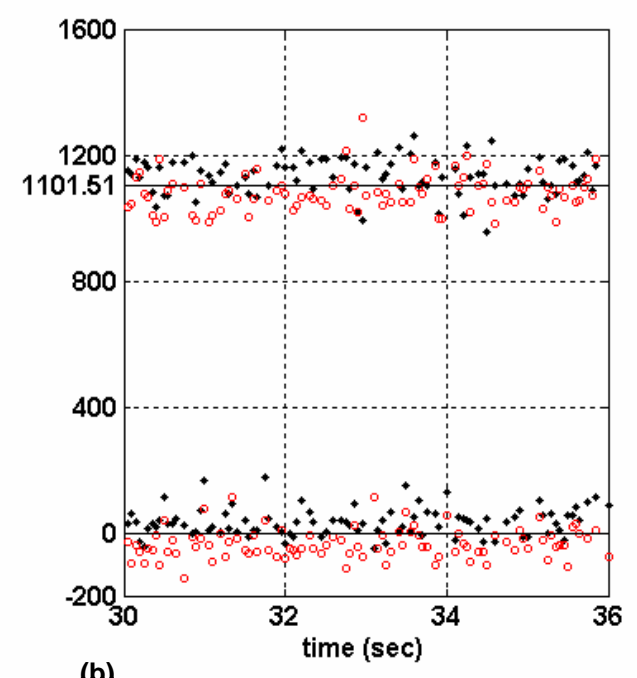

(b)

Figure 7. Simultaneous measurement of two components of velocity at two locations near the jet centerline of a Mach 1.6/Mach 5.5 enthalpy nearly perfectly-expanded jet: $(a)$, slightly off axis at $(x, y) / d=(0.879,0.175)$, and $(b)$, on axis at $(x, y) / d=(0.879,0.0)$. The axial component measurement is shown in the upper data plot, and the radial component in the lower data plot near zero velocity. The dotted line plot marked by a velocity label shows the CFD calculations corresponding to the measurement. 


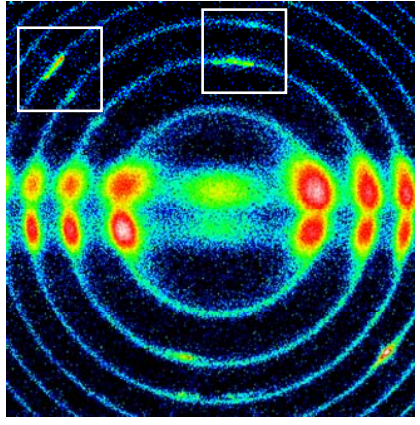

Figure 8. Instantaneous interferogram used to solve for two orthogonal velocity components showing the presence of (two) particles in the flow with no influence on the Rayleigh scattered light (showed by the horizontal patterns). calculation is independent of the Rayleigh signal itself. It was found that an equivalent of $30 \mathrm{~m} / \mathrm{sec}$ uncertainty in the measured FSR contributes to the standard deviation of the component being measured. This value is about 5 to 10 $\mathrm{m} / \mathrm{sec}$ higher than the values found during measurements performed in the laboratory in the same range of temperatures.

Although the experimental acoustic noise of the facility was substantial, as it will be described below, the simultaneous recording of the reference with the signal reduced the errors introduced by vibrations, detuning of the interferometer, and the laser to a minimal value.

A potential enhancement would be to control the intensity of the reference light source during measurements and to use more elaborate fitting techniques of the Rayleigh spectra, which would take into account the two-dimensional interferometer transfer function and the intensity distribution of the combined input beams. Another way to improve the fitting technique precision is to increase the signal (the number of photons scattered) by increasing the laser energy. Experiments at $165 \mathrm{~mJ} / \mathrm{pulse}$ and novel optical schemes in development, are designed to increase the interferogram resolution and the number of photons analyzed by the interferometer, and initial results have shown a decrease in these errors.

The small difference in the measured velocity values at these two locations demonstrates the system's capability for simultaneous measurement with high spatial resolution. In principle, the next outer fringe patterns can also be analyzed for velocity, but with reduced resolution. The average axial velocity over these two locations is about $2.5 \%$ different than the value obtained from the CFD calculations. The uncertainty in the location of the measurement may also contribute to this small difference of results.

It should be noted that the IRS system was located in close proximity, less than $1 \mathrm{~m}$ away from the jet flow having a very high level of noise $(\sim 140 \mathrm{~dB})$ and induced vibrations.

\section{B. The Influence of Noise, Vibrations, and Particles}

A brief description of how the facility influenced the IRS system during runs is summarized as follows. Two parts of the system were used to determine these influences: the Rayleigh camera, to monitor the beams fluctuations in the image plane (mostly observed on the vertical axis); and the CRB-View optics to determine the beam profiles, the overlap, and the stability of the beams at the measurement location. The facility water pump (required when the facility is run at high enthalpy), and the ventilation fan of the test cell were each started separately.

The largest number of rejected images were those containing dust particles either in the mixing layer of the jet and/or from particulates in the flow as well as when the measurements were performed in stagnant air (airborne dust particles). Some increase in the airborne dust that influences the interferogram rejection rate was observed when the facility ventilation fan was started. Fig. 8 shows two such dust particles in the upper half of the image (marked by white rectangles), and in the horizontally mirrored and vertically flipped bottom half of the image. As shown in this image, the disadvantage of using two viewing angles in the optical setup is the apparent doubling of the number of particles viewed by the CCD camera. This disadvantage is negligible in comparison with the big advantage of direct-viewing optics which allows for the selection of Rayleigh spectra from interferograms even when dust particles are present in the image. It is evident from this interferogram that the Rayleigh spectra from both beams are not contaminated with scattered light coming from these particles. 


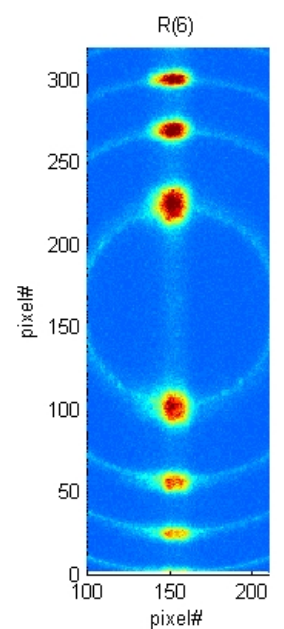

(a)

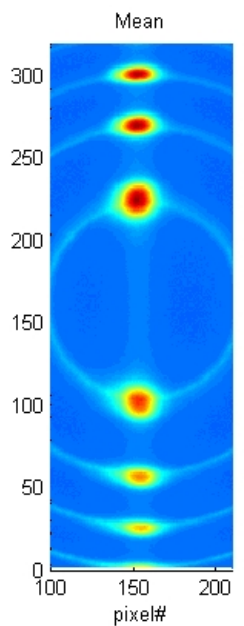

(b)

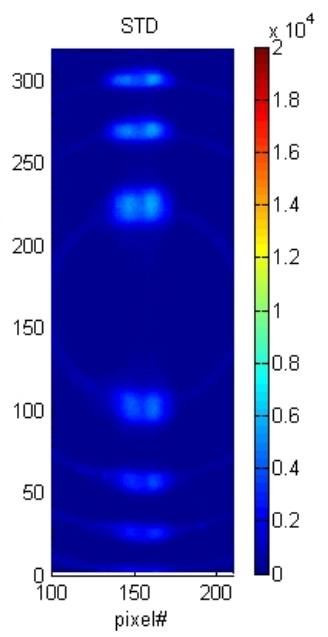

(c)
Figure 9. (a), single shot interferogram obtained in a large-scale supersonic jet used to determine the influence of vibrations on the IRS system. (b), the average of 78 measurements, and, (c), the standard deviation of intensity. The image is rotated clockwise $90^{\circ}$ from the original.

Referring to Fig. 9, the signal stability was investigated using interferograms that contain information to solve directly for one velocity component $\left(\mathrm{V}_{1}=\mathrm{v}\right)$ taken both in stagnant air and during the facility run. Again, the circular fringe pattern is generated by the laser frequency used as reference. The vertical dotted pattern is the image of the laser beam, rotated $90^{\circ}$ in the image, filtered through the interferometer. The diameter of the laser beam at the focusing location is about $100 \mu \mathrm{m}$. The initial position of the Rayleigh signal was set symmetrically around the center of the interferogram, as shown in the single shot interferogram in Fig. 9(a). The standard deviation for each pixel (Fig. 9(c)) from its mean (Fig. 9(b)) was calculated using 78 frames. The images containing light from dust particles were filtered out for simplicity.

The laser burst location in the image plane of the interferogram shows a variation of about one to two beam diameters in the raw images, and also is shown in Figs. 9(b-c) which have larger widths than the single shot image shown. This finding was critical in selecting the distance between the two parallel beams in the interferogram recording to avoid any spectral influence (spill-over) from the light coming from the other laser beam. Shot-to-shot variations of more than half FSR ( 2000 $\mathrm{m} / \mathrm{sec}$ ) of the interferogram location from its center were also found, making the measurement impossible without a system using referencing as used in the actual setup.

Very rarely, temporary failure of the injection seeding of the Nd:YAG laser was observed as a change in the wavelength and spectral profile (typically 1 or 2 frames from 1000 frames recordings). Also, a small wavelength drift of about 1 to $2 \mathrm{GHz}$ per hour depending on the room temperature was found when the laser was run few hours at full power. This drift could be compensated for by adjusting the external voltage applied to set the laser frequency. Since the interferogram fitting algorithm corrects for shot to shot variations in the laser's frequency, adjusting the laser frequency was performed approximately once per hour during the tests, as needed to maintain the frequency to within a few $\mathrm{GHz}$.

The spatial stability of the laser beams was investigated by monitoring the transverse intensity profiles of the laser beams near the focus using the CRB viewing system. The image presented in Fig. 10(a) shows the main green beam and the secondary green beams as they pass through the measurement region. The contour lines represent curves of constant intensity using an arbitrary intensity scale. The beam that arrives first at the measurement volume is of the highest intensity and is used for both the Rayleigh and the CARS measurement. The remaining green beams of

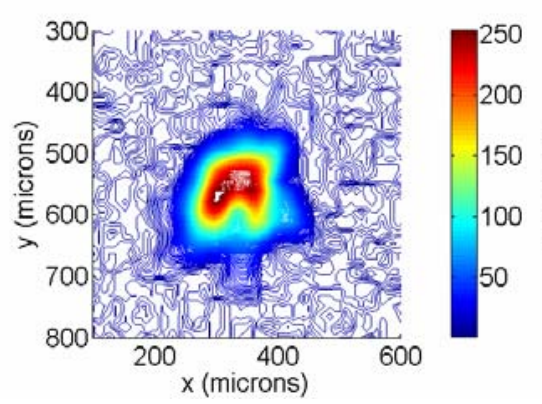

(a)

Figure 10. (a), The intensity profile of the laser burst before the facility run showing the overlapped collinear beams of the laser burst. The spatial location of the beams is in a plane transverse to the optical axis located at the center of the measurement volume. (b), and (c), the intensity profiles of the beams at two instances during the facility run.

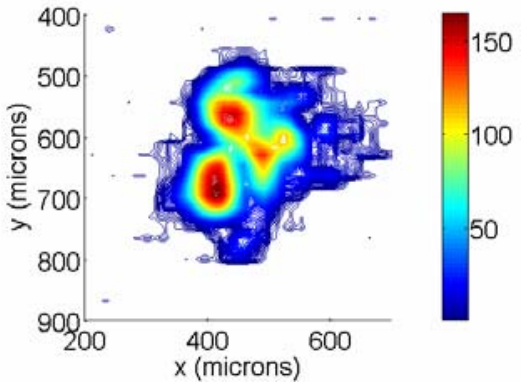

(b)

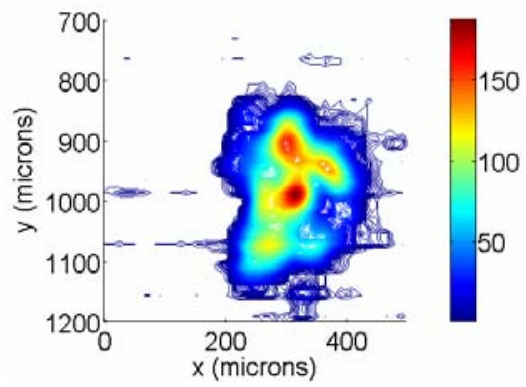

(c) 
decreasing intensity are the secondary collinear beams of the green laser burst used for Rayleigh scattering. Fig. 10(b) and Fig. 10(c) show the intensity profiles of the laser burst at two instances during the facility run. Notable are the apparent increase in the overall beam diameter and the change in intensity distribution of the laser beams as well as their separation and location. This is due to beam steering by optical component vibrations and flow density gradients. As a consequence of the beam fluctuations the number of photons per pixel in the image of the signal (horizontal patterns) is lower than during laboratory experiments. To compensate, an increase in the laser burst energy and more efficient collecting optics is required for these measurements at higher temperatures.

\section{Conclusions}

We described a measurement technique IRS that uses direct-viewing (as opposed to fiber) optics to collect, and one interferometer (solid etalon) to analyze elastically scattered laser light from molecules (Rayleigh scattering). We used an electron-multiplication CCD camera to capture the resulting interferograms. The laser source was formed using a pulse stretcher, generating a laser burst of multiple collinear beams, temporally separated and spread over a total of about $40 \mathrm{nsec}$, with at least three beams having more than $45 \mathrm{~mJ}$ per pulse. We demonstrated two methods of obtaining simultaneous single-shot measurements of two orthogonal components of velocity at multiple points in a large scale Mach 1.6 axisymmetric jet facility running at flow enthalpy specific to Mach 5.5 hypersonic flight.

The velocity was determined by analyzing single-shot interferograms, which contain the scattered light information from multiple collecting angles, the reference laser frequency, as well as the laser energy information. The technique used a new $(r, y)$ mapping and linearization to extract specific spectra, as well as a Gaussian approximation for the scattered spectra to calculate the spectral Doppler shift and the flow velocity. A comparison between $(r, \theta)$ and $(r, y)$ mapping methods of extracting the combined scattered and reference spectra was also performed.

Instantaneous two velocity components up to $1200 \mathrm{~m} / \mathrm{sec}$ were measured in the jet flow at multiple locations with a standard deviation between $36 \mathrm{~m} / \mathrm{sec}$ to $54 \mathrm{~m} / \mathrm{sec}$ at the highest flow temperature of about $1200 \mathrm{~K}$. The averaged measurement of the axial component of the jet agreed within 2.5\% of the CFD simulations using the VULCAN code based on a finite-volume discretization of the Favre-averaged Navier-Stokes equations. The minimum standard deviation of the measurement of about $8 \mathrm{~m} / \mathrm{sec}$ was obtained with the system in stagnant air at about $290 \mathrm{~K}$.

The collinear laser beams intensity and the overlap of the beams at the measurement location were monitored in real time using focal plane imaging (CRB viewing system). During the facility runs, the laser beams fluctuate and distribute spatially over more than one beam diameter, causing a reduction in the laser energy density at the measurement volume and a loss of signal intensity. Related to the previous laboratory work, the reduced signal to noise ratio and the uncertainty in locating the signal contributed to the higher standard deviation of these velocity measurements.

Dust particles did not affect the Rayleigh signal if the particles were imaged in interferograms far from the signal. When there is an increase in images that have particles present in most of the signal, as for example in the mixing layer flow and ambient air, there is a corresponding increase in the interferogram rejection rate. If the particles are partially contaminating the signal, it is still possible to extract the remaining spectra from portions of the interferogram not affected by them. Even a partial measurement compensates the disadvantage of not being able to solve the whole interferogram for multiple points or multiple velocity components.

In conclusion, we demonstrated velocity measurements in a high-speed high-enthalpy large-scale flow facility at NASA Langley Research Center using interferometric Rayleigh scattering (IRS). The measurement was made possible by: using direct viewing optics of Rayleigh scattered light to permit simultaneous measurements of twoorthogonal components of velocity at multiple points, minimizing the influence of dust particles and flow particulates; utilizing a solid etalon for spectral analysis; and referencing the laser wavelength with every measurement to compensate for the laser and the etalon variations due to facility noise.

The eventual outcome of this research will be to obtain experimental databases for improving the state of the art of CFD capabilities for predicting the effects of the test media on the flame-holding characteristics in hypersonic scramjet engines.

\section{Acknowledgments}

We thank Diego Capriotti, Tom Mills, and Barry Lawhorne and his team for their invaluable technical support during facility tests in DCSCTF at NASA LaRC. We recognize the contributions of Markus C. Weikl, Sarah Tedder, Gaetano Magnotti, Joseph W. Lee, Stephen B. Jones, and James Downey, during optical setup of the combined CARS-IRS system and during measurements. We also acknowledge Mark Kulick and Lloyd Wilson for fabricating special optical components. 
This work was supported by the NASA Fundamental Aeronautics Program - Hypersonics Project, Experimental Capabilities and Propulsion Disciplines and through a NASA Research Announcement grant from Experimental Capabilities. Also, the project was supported by George Rumford, program manager of the Defense Test Resource Management Center (DTRMC) Test and Evaluation/Science and Technology (T\&E/S\&T) program, under the Hypersonic Test focus area.

\section{References}

${ }^{1}$ J. Philip Drummond, Paul M. Danehy, Daniel Bivolaru, Richard L. Gaffney, Sarah A. Tedder, and Andrew D. Cutler, "Supersonic combustion research at NASA, 2007 Fall Technical Meeting," Eastern States Section of the Combustion Institute, University of Virginia, October 21-24, 2007.

2 A. D. Cutler, P. M. Danehy, R. R. Springer, S. O'Byrne, D. P. Capriotti, R. Deloach, "Coherent Anti-Stokes Raman Spectroscopic Thermometry in a Supersonic Combustor", AIAA Journal, Vol. 41, Num. 12, Dec. p. 2451-2459, (2003).

${ }^{3}$ Seasholtz, R. G., Zupanc, F. J. and Schneider, S. J., "Spectrally Resolved Rayleigh Scattering Diagnostics for HydrogenOxygen Rocket Plume Studies,” J. Propulsion and Power, Vol. 8, No. 5, 1992, pp. 935-942.

${ }^{4}$ Bivolaru, D, Danehy, P.M., Grinstead, K.D., Jr., Tedder, S., and Cutler, A.D., "Simultaneous CARS and Interferometric Rayleigh Scattering,” AIAA-2006-2968, 25th AIAA Aerodynamic Measurement Technology and Ground Testing Conference, San Francisco, CA, June 5-8, 2006.

${ }^{5}$ Bivolaru, D., Danehy, P. M., Lee, J. W., Gaffney, Jr. R. L., and Cutler, A. D., "Single-pulse Multi-point Multi-component Interferometric Rayleigh Scattering Velocimeter,” AIAA-2006-0836, 44th Aerospace Sciences Meeting, Reno, NV, January 9-12, 2006

6 S. O’Byrne, P. M. Danehy, S. A. Tedder, and A. D. Cutler, Dual-Pump Coherent Anti-Stokes Raman Scattering Measurements in a Supersonic Combustor AIAA Journal Vol. 45, No. 4, p. 922-933, April (2007).

${ }^{7}$ Bivolaru, D., Lee, J. W., Jones, S. B., Tedder, S., Danehy, P. M., Weikl, M. C., Magnotti, G., Cutler, A. D., "Mobile CARS - IRS Instrument for Simultaneous Spectroscopic Measurement of Multiple Properties in Gaseous Flows,” $22^{\text {nd }}$ International Congress on Instrumentation in Aerospace Simulation Facilities, Asilomar Conference Center, Pacific Grove, California, June 10 $-14,2007$.

${ }^{8}$ Miles, B., Richard, Lempert, R., Walter and Forkey, N. Joseph, "Laser Rayleigh Scattering,” Measurement Science and Technology, Vol. 12, pp. 33-51, 2001.

9 A. Mielke, K. Elam, and C. Sung, "Molecular Rayleigh Scattering Diagnostic for Dynamic Temperature, Velocity, and Density Measurements,” AIAA-2006-2969, 25th AIAA Aerodynamic Measurement Technology and Ground Testing Conference, San Francisco, California, June 5-8, 2006.

${ }^{10}$ P. L. Eggins and D. A. Jakson, “A two-component laser-Doppler anemometer using a single Fabry-Perot interferometer,” Journal of Physics D: Applied Physics 8, No. 4 (11 March 1975) L45-L47.

${ }^{11}$ Bivolaru, D., Danehy, P. M., and Lee, J. W, "Intracavity Rayleigh-Mie Scattering for multipoint, two-component velocity measurement,” Optics Letters, Vol. 31, No. 11, pp. 1645-1647, June, 2006.

${ }^{12}$ D. Bivolaru, G. C. Herring, "Focal-plane imaging of crossed beams in nonlinear optics experiments," Review of Scientific Instruments, 78, 056102 (2007).

${ }^{13}$ White, J. A. and Morrison, J. H., "A Pseudo-Temporal Multi-Grid Relaxation Scheme for Solving the Parabolized NavierStokes Equations," AIAA paper 99-3360, June, 1999. 